\title{
Obligation de signalement pour les mauvais traitements envers enfants
}

\author{
Virgile Woringer \\ Dr, ancien chef du Service de santé des écoles de la ville de Lausanne
}

Le Service de santé des écoles de la ville de Lausanne a mis en place dès 1989 entre les professionnels de l'école, enseignants, et les infirmières et assistants sociaux scolaires, médecins, psychologues et logopédistes scolaires, une procédure interne coordonnée de détection et de prise en charge des mauvais traitements. L'obligation légale cantonale de signaler est la simple application des principes exposés et de l'intention qui a prévalu à la rédaction du Code civil.

Cette question fait débat au niveau national depuis des décennies, périodiquement réactivée, vrai problème de santé publique toujours aigu, lancinant, pour l'instant sans solution durable, auquel une attention constante doit être portée en raison des dégâts personnels et sociaux que la maltraitance inflige à une partie de la population.

Le Service de santé des écoles de la ville de Lausanne a mis en place dès 1989 entre les professionnels de l'école, enseignants, et les infirmières et assistants sociaux scolaires, médecins, psychologues et logopédistes scolaires, une procédure interne coordonnée de détection et de prise en charge des mauvais traitements, en garantissant à chacun que les cas de maltraitance, éventuellement à signaler, le seraient sous la signature du médecin chef de service. Simultanément, une "permanence mauvais traitements», lieu de réunion non hiérarchisé, travaillant en réseau selon les règles habituelles, a été mis à disposition de ces professionnels ainsi que ceux des crèches et garderies lausannoises pour venir discuter des situations problématiques rencontrées dans l'exercice de leur activité, échanger les avis, et définir une action, que ce soit une prise de renseignements supplémentaire, une observation ou un signalement.

\section{La loi vaudoise sur la Protection des mineurs}

Le Code civil définit parfaitement le cadre d'intervention mais les lois cantonales varient. Historiquement, dans le canton de Vaud, la loi de 1978 définissait une obligation de signaler, mais sans règlement d'application, jusqu'à la promulgation d'une nouvelle loi en 2004 accompagnée d'un règlement d'application. Ce fut un changement dans la vision qu'avaient un certain nombre de professionnels de leur rôle, laissé dans ce domaine par l'ancienne loi de 1978 à leur seule appréciation, avec toute la variabilité imaginable.

La loi de 2004 précise qu'elle a pour but, entre autres, «d'assurer, en collaboration avec les parents, la protection et l'aide aux mineurs en danger dans leur développement, en favorisant l'autonomie et la responsabilité des familles», que «la responsabilité de

Un élément cardinal est de ne jamais rester seul avec son constat dans la phase d'évaluation initiale avant signalement.

pourvoir aux soins, à l'entretien et à l'éducation d'un mineur incombe en premier lieu aux parents", «toute décision prise en vertu de la présente loi doit l'être dans l'intérêt prépondérant du mineur», et que «le département est autorisé à échanger les informations ou données nécessaires à la prévention ou protection des mineurs avec les autorités concernées, dans les limites du respect dû à la sphère privée des intéressés.» Nous voilà rassurés en tant que médecins.

Les conditions d'intervention sont les suivantes: «Lorsque le développement physique, psychique, affectif ou social d'un mineur est menacé et que les parents ne peuvent y remédier seuls, le département prend les mesures de protection nécessaires", et que "ces mesures visent à prévenir, limiter ou faire disparaître le danger qui menace le mineur». L'aspect subsidiaire est ainsi parfaitement respecté, avec une 


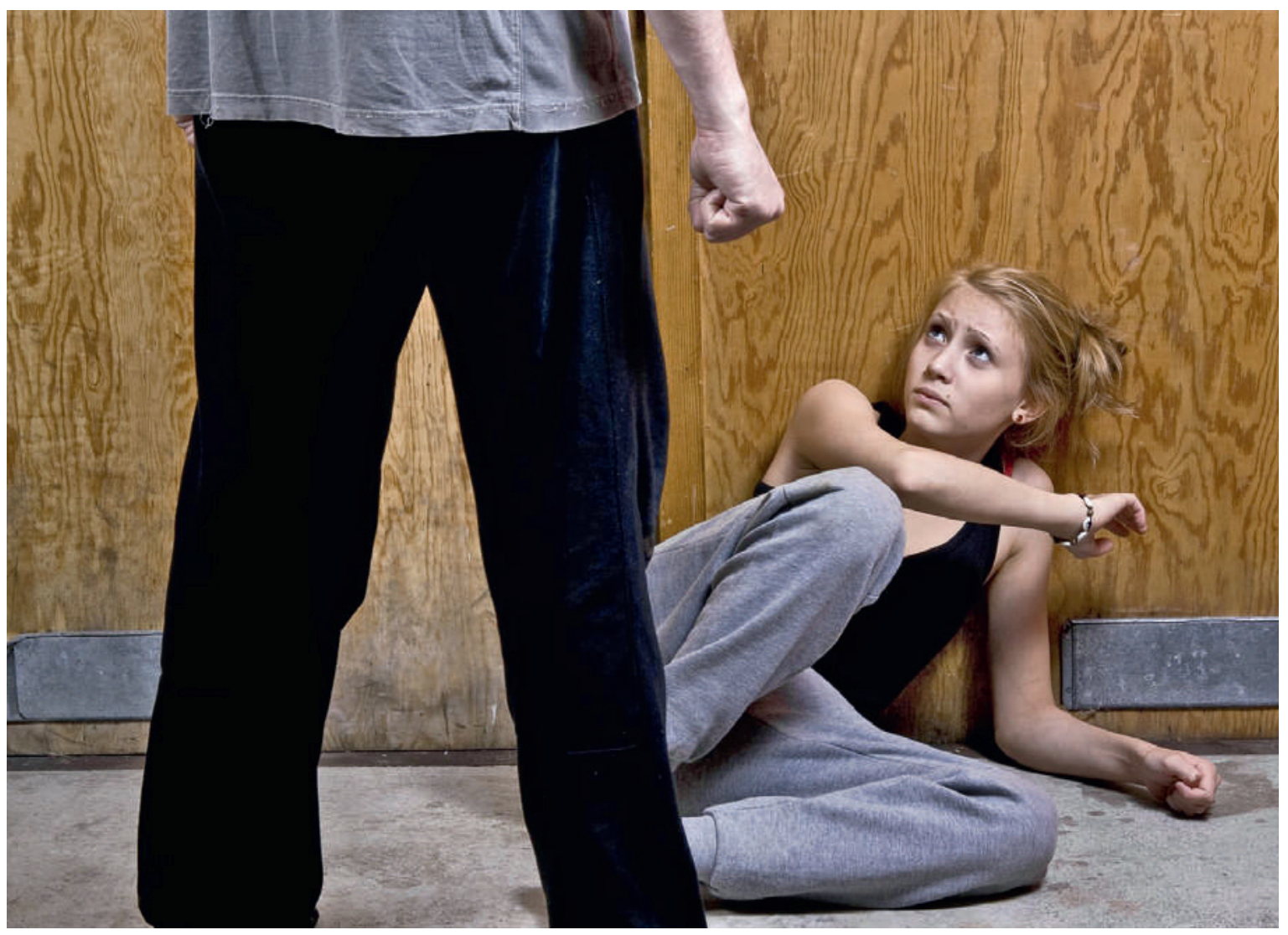

Pas seulement les enfants d'âge préscolaire sont concernés, mais aussi ceux de 7-15 ans.

action socio-éducative définie comme suit: «tout conseil, soutien ou aide apportés aux familles et mineurs en difficulté, ...», associant les parents: «lorsque le département intervient sans décision judiciaire, il met en œuvre l'action socio-éducative nécessaire d'entente avec les parents ou le représentant légal du mineur en danger dans son développement.» L'obligation particulière de signaler faite à ceux qui sont en rapport professionnel ou personnel avec des enfants complète le devoir exprimé au niveau fédéral. Mais cette obligation est précisée dans le règlement d'application de cette loi et s'articule autour des

La pratique régulière est le seul moyen de rester pleinement attentif, compétent, le plus adéquat dans les décisions prises.

connaissances et compétences des différentes personnes désignées: «..., la personne astreinte à l'obligation de signaler se réfère à la connaissance qu'elle a de la situation et à ses compétences professionnelles», avec un contenu clairement précisé en trois points absolument essentiels: «Le signalement doit porter sur les faits que la personne astreinte à l'obligation de signaler a observés, ce qui lui a été relaté et ce qu'elle en pense.» En cas de doute sur tout aspect de la situation dont elle a connaissance, la personne astreinte peut solliciter un conseil: «Toute personne visée par l'article 26 de la loi peut s'adresser au SPJ lorsqu'elle estime être confrontée à une situation de mise en danger du mineur dans son développement, notamment en cas de doute sur la démarche à entreprendre ou sur la nécessité de signaler.» Ce conseil est le plus souvent anonyme, et permet de se forger une opinion plus précise.

\section{Effets du changement de loi}

Les interventions socio-éducatives annuelles du Service de protection de la Jeunesse du canton de Vaud, en 1970 au nombre de 2271 (lieu de vie des mineurs pour $36 \%$ des situations dans la famille) (en 1990, $62 \%$ ), ont passé en 2000 à 3720, puis en 2005 (après la nouvelle loi) à 4942 (lieu de vie à $82 \%$ dans leur famille). Alors que le nombre des enfants suivis dans les autres lieux de vie que la famille n'a pas changé (environ 80o), celui des enfants suivis à domicile a ainsi évolué de manière très spectaculaire dès 2004 (avant, environ 3000, en 2013, 5700).

La statistique de l'âge des enfants concernés révèle quelques surprises: alors que l'on imagine que ce 
sont les enfants d'âge préscolaire qui sont les plus représentés, leur proportion est stable à 18-19\% depuis 1990! Par contre, celle des 7-15 ans a passé de $44 \%$ à $54 \%$ de 1990 à 2005 .

La maltraitance a-t-elle augmenté? Certainement pas, mais nous agissons de manière plus active.

\section{Position du médecin}

Je pense qu'une société ne fonctionne bien que si les personnes, dans les postes à responsabilité, que ce soit en matière professionnelle, politique, parentale, etc. assument pleinement leur charge. Les meilleurs dispositions, règlements, intentions se révèlent peu efficaces si ce socle n'est pas assuré. Le professionnel, quelles que soient ses connaissances, sa position sociale, son rapport à ses usagers, y est soumis comme tout autre, et la loi vaudoise ne fait que mettre en œuvre ces principes permanents. Ce devoir particulier de signaler paraît assez naturel, dans la mesure où la société doit pouvoir compter avec le concours de ceux

La statistique de l'âge des enfants concernés montre une augmentation des cas d'enfants de $7-15$ ans de $44 \%$ à $54 \%$.

qui sont particulièrement aptes à la détection des mauvais traitements en raison de leur position et de leurs compétences professionnelles. Ce devoir est d'ailleurs formalisé au niveau des CANTEAM des hôpitaux (depuis 1974). Pourquoi donc le refuser ailleurs?

Il convient de se demander quel est le degré de compétence qu'il convient d'atteindre pour faire correctement son travail. Un autre point incontournable pour le praticien est celui de la procédure à suivre, qui doit être apprise, comprise et exécutée avec une attention soutenue, et dont l'élément cardinal est de ne jamais rester seul avec son constat dans la phase d'évaluation initiale avant signalement. Cela implique que dans une situation de maltraitance, le travail en réseau implique d'en discuter avec des pairs, ou des collaborateurs formés/exercés. La loi vaudoise offre cette possibilité de conseil. Il faut réfléchir aux moyens pratiques d'agir de manière, le but de l'intervention étant d'abord de protéger l'enfant, puis de favoriser l'évolution de la situation vers une amélioration, en tentant de maintenir/rétablir le fonctionnement harmonieux des familles.

Compte tenu des éléments cités, il n'est pas certain que les cours dispensés dans les lieux de formation sont suffisants. La formation initiale est irremplaçable, mais comme dans tout travail en réseau, la pratique régulière est le seul moyen de rester pleinement attentif, compétent, le plus adéquat dans les décisions prises. Et comme chacun sait, le travail en réseau implique que l'on puisse être contredit, minorisé, sans que cela n'enlève au médecin ses responsabilités personnelles.

\section{Conclusion}

Pour moi, clairement, l'obligation légale cantonale de signaler est la simple application des principes exposés et de l'intention qui a prévalu à la rédaction du Code civil, et cela ne doit pas choquer mes confrères médecins. Chacun doit se conformer à la loi, et se demander s'il s'est mis dans la position d'être apte à le faire. Il n'y a aucune contradiction logique, conceptuelle, pour le médecin, qui vit en société, avec cette loi qui protège les plus faibles, et aussi les enfants maltraités contre leurs propres parents. La médecine et la religion ont historiquement été proches, sinon liées, jusqu'au haut Moyen Age. Le médecin n'a pas le pouvoir «d'absoudre». Si le seul devoir «moral» est affirmé dans la loi fédérale, sans dispositions légales contraignantes, le risque le plus prégnant est que l'on agisse de manière isolée, sans concertation, ce qui n'est pas bon et contraire à toute la pratique dans ce domaine. Se considérer comme un des acteurs nécessaires à une société saine est un devoir d'humilité: est-ce si difficile?

\footnotetext{
Références

- Les documents en ligne sur le site officiel du SPJ du canton de Vaud: www.vd.ch/themes/vie-privee/enfance-etjeunesse/protection-des-mineurs/
} 